Wright State University

CORE Scholar

Kno.e.sis Publications

The Ohio Center of Excellence in Knowledge-

Enabled Computing (Kno.e.sis)

$11-2007$

\title{
Supporting Complex Thematic, Spatial and Temporal Queries over Semantic Web Data
}

\author{
Matthew Perry \\ Wright State University - Main Campus \\ Amit P. Sheth \\ Wright State University - Main Campus, amit@sc.edu \\ Farshad Hakimpour \\ Prateek Jain \\ Wright State University - Main Campus
}

Follow this and additional works at: https://corescholar.libraries.wright.edu/knoesis

Part of the Bioinformatics Commons, Communication Technology and New Media Commons, Databases and Information Systems Commons, OS and Networks Commons, and the Science and Technology Studies Commons

\section{Repository Citation}

Perry, M., Sheth, A. P., Hakimpour, F., \& Jain, P. (2007). Supporting Complex Thematic, Spatial and Temporal Queries over Semantic Web Data. Lecture Notes in Computer Science, 4853, 228-246. https://corescholar.libraries.wright.edu/knoesis/722

This Conference Proceeding is brought to you for free and open access by the The Ohio Center of Excellence in Knowledge-Enabled Computing (Kno.e.sis) at CORE Scholar. It has been accepted for inclusion in Kno.e.sis Publications by an authorized administrator of CORE Scholar. For more information, please contact librarycorescholar@wright.edu. 


\title{
Supporting Complex Thematic, Spatial and Temporal Queries over Semantic Web Data*
}

\author{
Matthew Perry ${ }^{1}$, Amit P. Sheth ${ }^{1}$, Farshad Hakimpour ${ }^{2}$, Prateek Jain ${ }^{1}$ \\ ${ }^{1}$ Kno.e.sis Center, Department of Computer Science and Engineering, Wright State \\ University, Dayton, OH, USA \\ ${ }^{2}$ LSDIS Lab, Department of Computer Science, University of Georgia, Athens, GA, USA \\ \{perry.66, amit.sheth, jain.18\}@wright.edu, \{fhakimpour\}@uga.edu
}

\begin{abstract}
Spatial and temporal data are critical components in many applications. This is especially true in analytical domains such as national security and criminal investigation. Often, the analytical process requires uncovering and analyzing complex thematic relationships between disparate people, places and events. Fundamentally new query operators based on the graph structure of Semantic Web data models, such as semantic associations, are proving useful for this purpose. However, these analysis mechanisms are primarily intended for thematic relationships. In this paper, we describe a framework built around the RDF metadata model for analysis of thematic, spatial and temporal relationships between named entities. We discuss modeling issues and present a set of semantic query operators. We also describe an efficient implementation in Oracle DBMS and demonstrate the scalability of our approach with a performance study using a large synthetic dataset from the national security domain.
\end{abstract}

Keywords: Ontology, Semantic Analytics, RDF Querying, Spatial RDF, Temporal RDF

\section{Introduction}

Analytical applications are increasingly exploiting complex thematic relationships between named entities as a powerful tool in the analysis process. Such "connecting the dots" applications are common in many domains, for example national security, drug discovery and medical informatics. Semantic Web data models, such as Resource Description Framework (RDF) [1], fit nicely with this analysis paradigm because relationships are modeled as first class objects. Fundamentally new analytical operators based on the graph structure of RDF have emerged (e.g., semantic associations [2] and subgraph discovery [3]) which allow querying for complex relationships between named entities where an ontology provides the context or domain semantics. We use the term semantic analytics to refer to this process of searching, analyzing and visualizing semantically meaningful connections between named entities. Many successful applications of semantic analytics can be seen in the

* This work is partially funded by NSF-ITRIDM Award \#0325464 \& \#0714441 entitled "SemDIS: Discovering Complex Relationships in the Semantic Web." 
literature (e.g., identifying conflict of interest [4], detecting patent infringement [5] and metabolic pathway discovery [6]).

While spatial and temporal data often play a crucial role in many analytical domains, research in semantic analytics has focused on thematic relationships. Current approaches do not adequately handle spatial and temporal data. Furthermore, traditional spatial and spatiotemporal data models used for GIS [7] excel at modeling and analyzing spatial and temporal relationships between geographic entities but tend to model the thematic aspects of a given domain as directly attached attributes of geospatial entities.

In a recent work [8], we have tried to overcome this limitation by modeling spatial, temporal and thematic data using ontologies and temporal RDF graphs [9]. An upperlevel ontology is used to define the basic classes and relationships of the thematic and spatial domains. With this approach, thematic entities and relationships are represented as first class objects and are modeled separately from their spatial properties (basic spatial features, such as points and lines, termed spatial entities). Thematic entities and events are connected to spatial entities through located_at and occurred_at relationships modeled in the upper-level ontology. Deeper domain ontologies are integrated with this upper-level ontology through $r d f$ s:subClass $O f$ and rdfs:subProperty $O f$ statements. A unique aspect of this approach is that a 1-to-1 mapping between thematic and spatial entities is not enforced. Rather, a many-tomany mapping is achieved by utilizing indirect thematic connections (specified with domain ontologies) between entities. For example, using a military ontology, a soldier could be associated with the spatial properties of his residence through one set of relationships (Soldier - lives_at - Residence - located_at - Spatial_Entity) or with the locations of his training facilities using a different set of relationships (Soldier member_of-Military_Unit-trains_at-Base - located_at-Spatial_Entity).

A variety of query operators are possible over this model which combine thematic relationships with spatial and temporal relationships, thus adding more expressive domain semantics to spatial and temporal queries. We argue that by incorporating more complex models and operators for thematic data, a GIS can be significantly more useful in applications which require complex thematic analysis in addition to spatial and temporal analysis.

Spatial and temporal data bring many unique challenges to semantic analytics applications. Thematic relationships can be explicitly stated in the RDF graph, but some spatial and temporal relationships (e.g., quantitative relationships like distance) are implicit and only evident after additional computation. RDF and RDF Schema (RDFS) inferencing rules [10,11] are also affected as the temporal properties of asserted statements will have implications on the temporal properties of the corresponding inferred statements.

Example (biochemical threat detection): Suppose an intelligence analyst is assigned the task of monitoring the health of soldiers in order to detect possible exposure to a chemical or biological agent which may imply a biochemical attack. In this case, the analyst may search for relationships connecting a sick soldier to potential chemical or biological agents by matching the soldier's symptoms with known reactions to these agents. In addition, the analyst could further determine the likelihood of a particular chemical substance by querying for associations between the substance and enemy groups in the knowledgebase. For example, a member of the 
group may have worked at a facility which was reported to have produced the chemical. It is doubtful that such an analysis could produce definitive evidence of a biochemical attack, but incorporating spatial and temporal relationships could help in this regard. For instance, the analyst may want to limit the results to soldiers and enemies in close spatial proximity (e.g., find all soldiers with symptoms indicative of exposure to chemical $X$ which fought in battles within 2 miles of sightings of any members of enemy group $Y$ ). We may pose the following SQL query involving the spatial_eval table function for such a search:

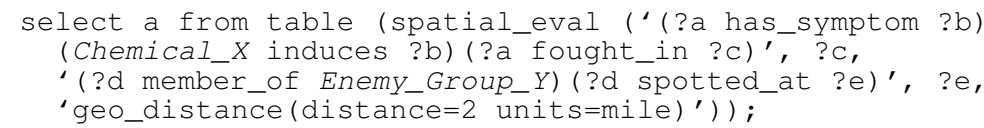

With this query, we are using the spatial_eval operator to specify (1) a relationship between a soldier, a chemical agent and a battle location and (2) a relationship between members of an enemy organization and their known locations. We are then limiting the results based on the spatial proximity of the battles and enemy sightings. Additionally, we provide a spatial_extent operator which allows retrieving the spatial geometry associated with the spatial entities composing a thematic relationship and optionally filtering the results using a spatial predicate. For example, find all soldiers participating in military events that take place within an input bounding box. For temporal aspects, we provide an analogous temporal_extent operator which returns the temporal properties of a given relationship and allows optional filtering. For example, return all soldiers exhibiting a given symptom during a specific time period. We also provide a temporal_eval operator which can answer queries such as find soldiers who exhibited symptoms after participating in a given military event.

This paper focuses on providing a framework to support spatial and temporal analysis of RDF data. RDF is a World Wide Web Consortium (W3C) standard for representing ontologies and corresponding instance data. We address problems of both data storage and operator design and implementation. Specifically, the contributions of this paper are:

- A storage and indexing scheme for spatial and temporal RDF data

- An efficient treatment of temporal RDFS inferencing

- The definition and implementation of four spatial and temporal query operators

- A performance study using a large RDF dataset

The remainder of the paper is organized as follows. Section 2 discusses background information and related work regarding data modeling and querying. Section 3 further describes the set of spatial and temporal query operators. Section 4 describes the implementation of this framework in Oracle DBMS. An experimental evaluation of this implementation follows in Section 5, and Section 6 gives conclusions.

\section{Background and Related Work}

In this section, we discuss background information and related work with regards to data modeling and querying Semantic Web data. 
RDF and Ontologies. RDF [1] has been adopted by the W3C as a standard for representing metadata on the Web. Resources in RDF are identified by Uniform Resource Identifiers (URIs) that provide globally-unique and resolvable identifiers for entities on the Web. These resources are described through participation in relationships. Relationships in RDF are called Properties and are binary relationships connecting resources to other resources or resources to Literals, i.e., literal values such as Strings or Numbers. These binary relationships are encoded as triples of the form (Subject, Property, Object), which denotes that a resource - the Subject - has a Property whose value is the Object. These triples are referred to as Statements. RDF also allows for anonymous nodes called Blank Nodes which can be used as the Subject or Object of a statement. We call a set of triples an RDF graph, as RDF data can be represented as a directed, labeled graph with typed edges and nodes. In this model, a directed edge labeled with the Property name connects the Subject to the Object.

RDF Schema (RDFS) [10] provides a standard vocabulary for describing the classes and relationships used in RDF statements and consequently provides the capability to define ontologies. Ontologies serve to formally specify the semantics of RDF data so that a common interpretation of the data can be shared across multiple applications. RDFS allows us to define hierarchies of class and property types, and it allows us to define the domain and range of property types.

Additionally, a set of entailment rules are defined for RDF and RDFS [11]. These rules essentially specify that an additional triple can be added to the RDF graph if the graph contains triples of a specific pattern. Such rules describe, for example, the transitivity of the $r d f s$ :subClass $O f$ property.

Temporal RDF Graphs. In order to analyze the temporal properties of relationships in RDF graphs, we need a way to record the temporal properties of the statements in those graphs, and we must account for the effects of those temporal properties on RDFS inferencing rules. For this purpose, we adopt temporal RDF graphs defined in [9]. Temporal RDF graphs model absolute time and are defined as follows. Given a set of discrete, linearly ordered time points $T$, a temporal triple is an RDF triple with a temporal label $t \in T$. A statement's temporal label represents its valid time. The notation $(s, p, o):[t]$ is used to denote a temporal triple. The expression $(s, p, o):\left[t_{1}\right.$, $\left.t_{2}\right]$ is a notation for $\left\{(s, p, o):[t] \mid t_{1} \leq t \leq t_{2}\right\}$. A temporal RDF graph is a set of temporal triples. For example, consider a soldier $s 1$ assigned to the $1^{\text {st }}$ Armored Division $\left(1^{s t} A D\right)$ from April 3, 1942, until June 14, 1943, and then assigned to the $3^{\text {rd }}$ Armored Division $\left(3^{r d} A D\right)$ from June 15,1943 , until October 18,1943 . This would yield the following triples: ( $\left(1\right.$, assigned_to, $\left.1^{s t} A D\right)$ : [04:03:1942, 06:14:1943], $(s 1$, assigned_to, $\left.3^{r d} A D\right)$ : [06:15:1943, 10:18:1943]. Any temporal ontology that defines a vocabulary of time units can be used to precisely specify the start and end points of time intervals.

As discussed in [9], we must account for the effects of temporal labels on RDFS inferencing rules. To incorporate inferencing into temporal RDF graphs, we must use a basic arithmetic of intervals to derive the temporal label for the inferred statements. For example, interval intersection would be needed for $r d f s$ :subClassOf (e.g., ( $x$, 
rdfs:subClassOf, y) : [1, 4] $\wedge(y, r d f s: s u b C l a s s O f, z):[3,5] \rightarrow(x, r d f s: s u b C l a s s O f$, z) : $[3,4])$.

Related Work. We will first discuss our modeling approach using temporal RDF as it compares with other spatiotemporal models in the literature. For a recent survey, see [7]. Of the models discussed in the literature, the object-oriented and event-based models and the three domain model are most similar to our RDF-based approach. The three domain model, introduced by Yuan, is described in [12, 13]. This model represents semantics, space and time separately. To represent spatiotemporal information in this model, semantic objects are linked via temporal objects to spatial objects. This provides temporal information about the semantic (thematic) properties of a given spatial region. This is analogous to temporal located_at and occurred_at relationships in our model. The three domain model is quite similar to our approach in that it represents thematic entities as first class objects rather than attributes of geospatial objects. The key difference is that the three domain model relies on direct connections from thematic entities to spatial regions whereas our model allows indirect connections composed of sequences of thematic relationships, which is made possible by a richer modeling of the thematic domain. Additionally, relaxing the direct connection requirement better tolerates incompleteness of information - a necessity when handling Web data. In [14], the authors discuss a combination of the object-oriented and event-based modeling approaches for dynamic geospatial domains. They define an upper-level ontology similar to the one we present in [8]. They model the concept of a setting and a situate function which maps entities and events to settings. Settings can be spatial, temporal, or spatiotemporal. In contrast to our work, the authors focus on geospatial objects and events and model what we would consider a thematic entity (e.g., an airplane) as a geospatial entity. That is, the separation between the thematic and spatial domains is not as strongly emphasized. Our RDF-based modeling approach provides a means to assign spatial properties to those entities not directly connected to a spatial setting and allows deeper analysis of purely thematic relationships.

Many RDF query languages have been proposed in the literature. These include SQL-like languages (e.g., SPARQL [15]), functional languages (e.g., RQL [16]), rulebased languages (e.g., TRIPLE [17]) and graph traversal languages (e.g., RxPath [18]). Efficient implementations of these languages for persistent RDF data usually involve translation into a SQL query against an underlying RDBMS representation of the RDF data (e.g., Jena2 [19], RDFSuite [20]). As an alternative to defining a new query language, an approach for querying RDF data directly in SQL has been proposed [21]. This facilitates easy integration with other SQL queries against traditional relational data and saves the overhead of translating data from SQL to the RDF query language data format. Our implementation follows this approach and introduces new SQL functions for spatial and temporal querying of RDF data.

Work is somewhat limited with regards to incorporating spatial and temporal relationships into queries over Semantic Web data. Examples of querying geospatial RDF data are mostly seen in web applications and semantic geospatial web services $[22,23]$ in the spirit of the Geospatial Semantic Web [24]. In general, query processing proceeds by translating RDF representations of spatial features into geometric representations on the fly and then performing spatial calculations, and the 
focus is more on interoperability than efficient query processing. The SPIRIT spatial search engine [25] combines an ontology describing the geospatial domain with the searching and indexing capability of Oracle Spatial for the purposes of searching documents based on the spatial features associated with named places mentioned in the document. In contrast, our searching operators are intended for general purpose querying of ontological and spatial relationships. Querying for temporal data in RDF graphs is less complicated as RDF supports typed literals such as xsd:date, and corresponding query languages support filtering results based on literal values. However, this is far from supporting full temporal RDF as graphs discussed in this paper. In addition to formally defining temporal RDF graphs, Gutierrez et al. briefly discussed aspects of a query language for these graphs, but no implementation issues were mentioned [9]. Also, to the best of our knowledge, this paper is the first to investigate implementation of RDFS inferencing which incorporates the concept of valid time for RDF statements.

\section{Query Operators}

In this section, we introduce a set of spatial and temporal query operators for searching and analyzing spatial and temporal relationships between named entities in temporal RDF graphs. These operators are an adequate functional set in that they (1) allow precise specification of a thematic portion of the RDF graph (subgraph), (2) provide facilities to compute spatial and temporal properties of these subgraphs and (3) allow filtering and joins based on the computed spatial and temporal properties. The operators are implemented as SQL table functions. Table functions produce a set of rows as output which can be queried. They are used in SQL queries in the same manner as a database table name. For example, we may have the query select $x$, y from table (table_func $(\ldots)$ ) order by $x$.

Graph Patterns. SPARQL-like graph patterns are the basic building block of these operators. Intuitively, a graph pattern is a set of RDF triples where the subjects, properties and/or objects may be replaced with variables. In general, a graph pattern query against an RDF graph $G$ returns a set of mappings between the variables in the graph pattern and terms (URIs, Blank Nodes and Literals) in $G$ such that substituting the mapped terms into the graph pattern results in a set of triples actually present in $G$. We refer to the set of triples resulting from a substitution as a graph pattern instance, and the result of a graph pattern query on a given RDF graph $G$ is the set of variable bindings for all matching graph pattern instances in $G$. Fig. 1 illustrates these concepts for an example graph pattern query.

Spatial Query Operators. We define two spatial query operators for RDF graphs containing geospatial data: spatial_extent and spatial_eval. The following descriptions assume the existence of a class Geometry in the ontology which models spatial objects, and we use the term spatial feature to refer to an SDO_GEOMETRY object that would be stored in Oracle Spatial (i.e., the implementation of Geometry). 


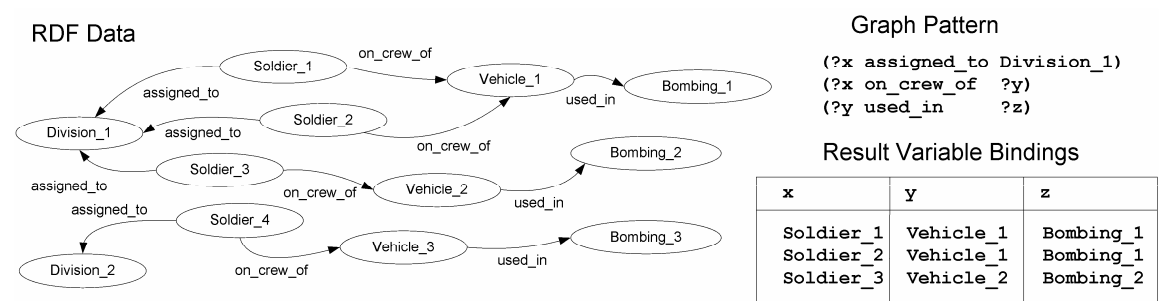

Fig. 1. Example graph pattern with resulting variable bindings.

The first spatial operator, spatial_extent, is intended to retrieve the spatial feature of the Geometry connected to a thematic entity and optionally filter the results based on the properties of the spatial feature. The signature for the corresponding table function is shown below:

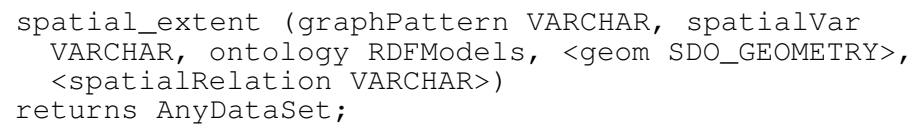

The graphPattern parameter specifies the relationship between a thematic entity and a Geometry, for example (Soldier, fought_in, Battle) (Battle, located_at, Geometry). The spatialVar parameter identifies the variable in the graph pattern that corresponds to a Geometry, and ontology determines the ontology to search against. This function returns a table with rows containing columns for each variable in the graph pattern and one column for the spatial features. Each row contains the URI bound to each variable and the spatial feature corresponding to the Geometry bound to spatialVar (displayed as well known text format in Oracle). Two optional parameters, a spatial feature and a spatial relationship, can be used to filter the graph pattern instances. In this case, the table would only contain those graph pattern instances whose associated spatial features satisfy the specified spatial relation with the input spatial feature. We support the following spatial relationships: touch, overlap, equal, inside, covered by, contains, covers, any interact and within distance.

The second spatial operator, spatial_eval, acts as a spatial join between graph pattern instances. It is intended to allow for searching thematic entities based on their spatial relationships. The signature for the corresponding table function is shown below:

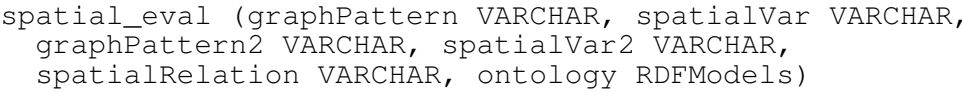
graphPattern 2 and spatialVar2 specify the right hand side. spatialRelation identifies the spatial join condition. This function returns a table containing a column for each variable in graphPattern and graphPattern 2 and a column for each associated spatial feature ( $s f 1$ and $s f 2$ ). For each row in the resulting table, $s f 1$ spatialRelation $s f 2$ evaluates to true.

Temporal Query Operators. We define two temporal query operators for temporal RDF graphs: temporal_extent and temporal_eval. The basic idea behind the operators 
is that we compute a temporal interval for a graph pattern instance based on the temporal properties of the triples making up the graph pattern instance.

The first temporal operator, temporal_extent, is used to compute the temporal interval for a graph pattern instance and optionally filter the results based on the computed temporal interval. We support two basic intervals for a graph pattern instance: the interval during which the entire graph pattern instance is valid (INTERSECT) and the interval during which any part of the graph pattern is valid $(R A N G E)$. The signature for the corresponding table function is shown below.

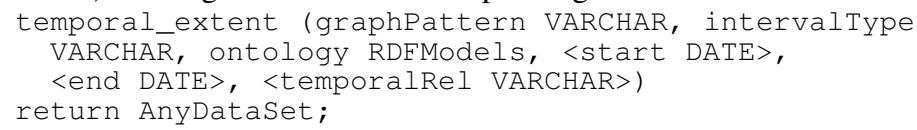

This function takes three parameters as input, specifically a graph pattern, a String value specifying the interval type (INTERSECT or RANGE), and a parameter specifying the ontology to search against. The table returned contains a column for each variable in the graph pattern and two DATE columns which specify the start and end of the time interval computed for the graph pattern instance. Three optional parameters, two DATE values to identify the boundaries of a time interval and a temporal relationship, can be used to filter the found graph pattern instances. In this case, assuming the DATE columns in the returned table are named stDate and endDate, each row in the result satisfies the condition [stDate, endDate] temporalRel [start, end]. We currently support seven temporal relationships: before, after, during, overlap, during_inv, overlap_inv and any interact.

The second temporal operator, temporal_eval, acts as a temporal join operator for graph pattern instances. The corresponding table function has the following signature:

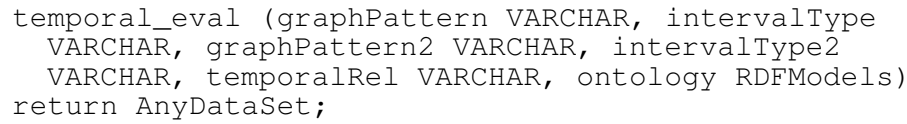

graphPattern and intervalType specify the left hand side of the join operation, while graphPattern 2 and intervalType 2 specify the right hand side. temporalRel identifies the join condition. This function returns a table containing a column for each variable in graphPattern and graphPattern2 and four DATE columns (start1, end1, start2, end2) to indicate the time interval for each found graph pattern instance. For each row in the resulting table, [start1, end1] temporalRel [start2, end2] evaluates to true.

\section{Implementation in Oracle}

In this section, we describe the implementation of our spatial and temporal RDF query operators in Oracle DBMS. The implementation builds upon Oracle's existing support for RDF storage and inferencing and support for spatial object types and indexes. We create SQL table functions for each of the previously discussed query operators. Additional structures are created to allow for spatial and temporal indexing of the RDF data for efficient query evaluation. We should note that in general this approach is not limited to Oracle and could be implemented using any extensible DBMS that supports user-defined object types and functions. 

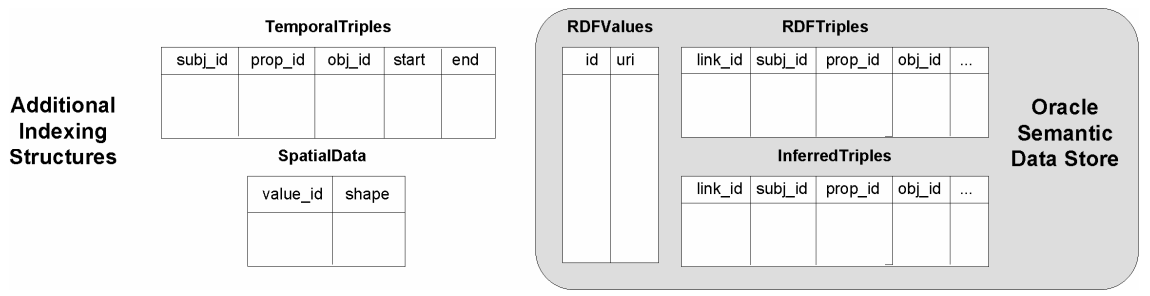

Fig. 2. Storage structures for RDF data. Existing tables of Oracle Semantic Data Store are shown on the right, and our additional tables for efficiently searching spatial and temporal data are shown on the left.

Existing Oracle Technologies. Oracle's Semantic Data Store [26] provides the capabilities to store, infer, and query semantic data, which can be plain RDF descriptions and RDFS based ontologies. To store RDF data, users create a model (ontology) to hold RDF triples. The triples are stored after normalization in two tables: an RDFValues table which stores RDF terms and a numeric id and an RDFTriples table which stores the ids of the subject, predicate and object of each statement. Users can optionally derive a set of inferred triples based on user-defined rules and/or RDFS semantics. These triples are materialized by creating a rules index and stored in a separate InferredTriples table. These storage structures are illustrated in Fig. 2. A SQL table function is provided that allows issuing graph pattern queries against both asserted and inferred RDF statements.

Oracle Spatial [27] provides facilities to store, query and index spatial features. It supports the object-relational model for representing spatial geometries. A native spatial data type, SDO_GEOMETRY, is defined for storing vector data. Database tables can contain one or more SDO_GEOMETRY columns. Oracle Spatial supports spatial indexing on SDO_GEOMETRY columns, and provides a variety of procedures, functions and operators for performing spatial analysis operations.

Data Representation. Our Framework supports spatial and temporal data serialized in RDF using an RDFS ontology discussed in [28]. This ontology models the concept of a Geometry Class and allows for recording coordinate system information and representing points, lines, and polygons. This model complies with the OGC simple feature specification [29]. Using this representation, spatial features are stored as instances of Geometry and are uniquely identified by their URI. Temporal labels are associated with statements using RDF reification, as suggested in [9]. Reification allows us to assert statements about RDF statements. Our framework supports time interval values serialized as instances of the Class Interval from this ontology. A property type, temporal, is defined to assert that a statement has a valid time which is represented as an Interval instance.

Indexing Approach. In order to ensure efficient execution of graph pattern queries involving spatial and temporal predicates, we must provide a means to index portions of the RDF graph based on spatial and temporal values. Basically, this is done by building a table mapping Geometry instance URIs to their SDO_GEOMETRY representation and by building a modified RDFTriples table which also stores the 
temporal intervals associated with the triple. In order to build these indexes, users first load the set of asserted RDF statements into Oracle Semantic Data Store and build an RDFS rules index.

Spatial Indexing Scheme. We provide the procedure build_geo_index() to construct a spatial index for a given ontology. This procedure first creates the table SpatialData (value_id NUMBER, shape SDO_GEOMETRY) for storing spatial features corresponding to instances of the class Geometry in the ontology. value_id is the id given to the URI of the Geometry instance in Oracle's RDFValues table, and shape stores the SDO_GEOMETRY representation of the Geometry instance (see Fig. 2). This table is filled by querying the ontology for each Geometry instance, iterating through the results and creating and inserting SDO_GEOMETRY objects into the spatial indexing table. Finally, to enable efficient searching with spatial predicates on this table, a spatial (R-Tree) index is created on the shape column.

Temporal Indexing Scheme. Our temporal indexing scheme is a bit more complicated, as it must account for temporal labels on statements inferred through RDFS semantics. However, we only need to handle a subset of the RDFS inference rules. This is the case because we are not interested in handling temporal evolution of the ontology schema. What we need to handle are temporal properties of instance data. Specifically, we need to account for temporal labels of inferred rdf:type statements and statements resulting from rdfs:subPropertyOf statements. rdf:type statements result from the following rules: (1) ( $x$, rdf:type, $y) \wedge(y$, rdfs:subClass $O f, z) \rightarrow(x$, $r d f:$ type, $z)$, and (2) $(x, p, y) \wedge(p, r d f s:$ domain, $a) \wedge(p, r d f s:$ range, $b) \rightarrow(x$, rdf:type, $a),(y, r d f: t y p e, b)$. We infer instance statements from $r d f$ s:subProperty $O f$ using the following rule: (1) $(x, p, y) \wedge(p, r d f s:$ subProperty $O f, q) \rightarrow(x, q, y)$. In each case, if we assume that schema level statements in the ontology are eternally true, the temporal label of an inferred instance statement $s$ is the union of the time intervals of all statements which can be used to infer $s$.

We provide the procedure build_temporal_index() to create a temporal index for a given ontology. This procedure executes in three phases.

The first phase creates the temporary table asserted_temporal_triples (subj_id NUMBER, prop_id NUMBER, obj_id NUMBER, start DATE, end DATE). The ontology is then queried to retrieve all asserted temporal reifications. The subject, property, and object ids of each temporally reified statement and the start time and end time are inserted into this temporary table. The final step of this phase inserts statements without asserted temporal reifications into the asserted_temporal_triples table using min_start_time and max_end_time as the default start and end times. These values are specified during index creation. Additionally, all schema-level statements also receive these start and end values to denote that the ontology schema is always valid.

At this point, we have recorded the temporal values for each asserted statement, and the second and third phases perform the temporal inferencing process and create the final temporal triples table (see Fig. 2). In the procedure TemporalInference (shown below), we first create a second temporary table redundant_triples (subj_id NUMBER, prop_id NUMBER, obj_id NUMBER, start DATE, end DATE). Then, we iterate through the asserted_temporal_triples table and add any inferred statements to 
the redundant_triples table. In this step, the temporal label of the asserted statement is directly assigned to the corresponding inferred statements. This procedure results in possibly redundant and overlapping intervals for each statement, so a third phase iterates through this table and cleans up the time intervals for each statement. The cleanup phase first sorts redundant_triples by (subj_id, prop_id, obj, start_id) and then makes a single pass over the sorted set to merge overlapping intervals for statements with the same subject, property and object. The final result of this process is a table TemporalTriples (subj_id NUMBER, prop_id NUMBER,obj_id NUMBER, start DATE, end DATE) which contains the complete set of asserted and inferred temporal triples (see Fig. 2).

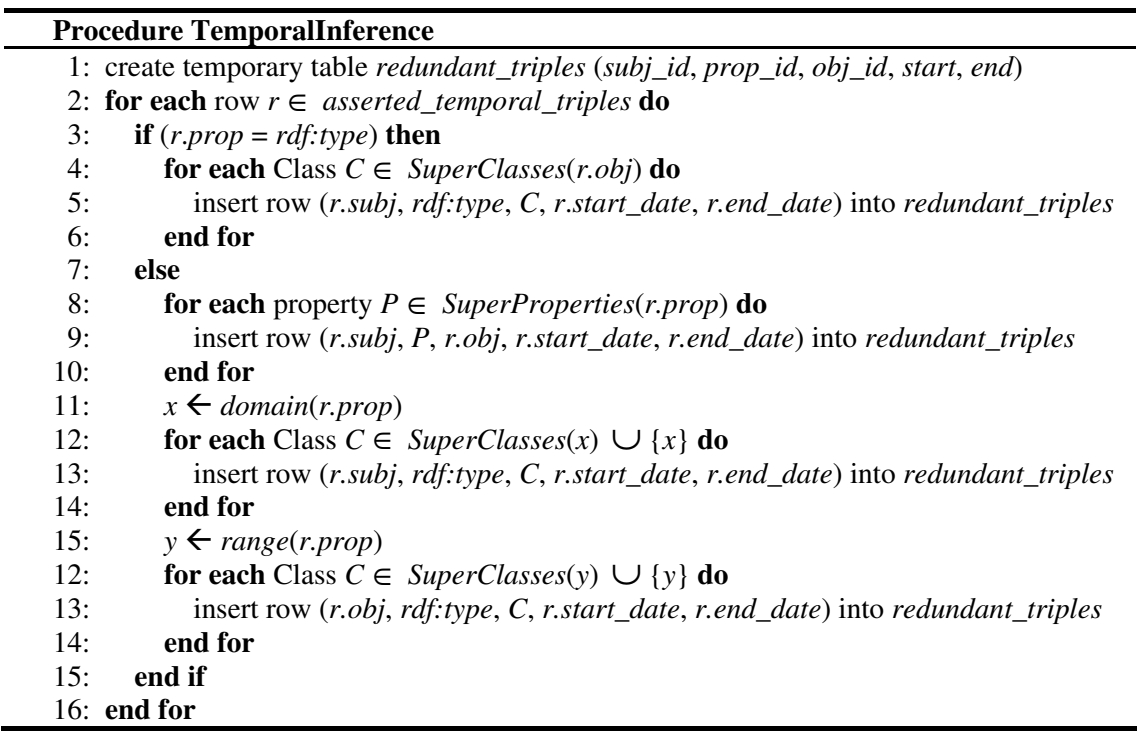

Operator Implementation. The SQL table functions were implemented using Oracle's ODCITable interface methods [30]. With this scheme, users implement a $\operatorname{start}(), \operatorname{fetch}()$ and $\operatorname{close}()$ method for the table function. Generally, in the start() method, table function parameters are parsed, and a SQL query is prepared and executed against the underlying database tables. The $f e t c h()$ method fetches a subset of rows from the prepared query and returns them. The $f e t c h()$ method is invoked as many times as necessary by the kernel until all result rows are returned. The close() method performs cleanup operations after the last $f e t c h()$ call.

Each of the table functions takes a graph pattern and ontology as input. In $\operatorname{start}($ ), the graph pattern is parsed and transformed into a self-join query against the TemporalTriples table corresponding to the input ontology. We will illustrate this process with the following example: (?a on_crew_of ?b) (?b used_in ?c)

First, URIs in the graph pattern are resolved to numeric ids through a lookup in the RDFValues table. Assume that in this case the ids of member_of and used_in are 1 and 2 respectively. Next, we perform a self join of the TemporalTriples table with two sets of conditions in the where clause: (1) we must restrict the rows of each table based on the ids of the URIs in the graph pattern and (2) we must create a join 
condition based on variable correspondences between different parts of the graph pattern. We must also join with the RDFValues table to resolve the ids of URIs bound to variables to actual URI Strings for return from the function. The graph pattern above results in the following query:

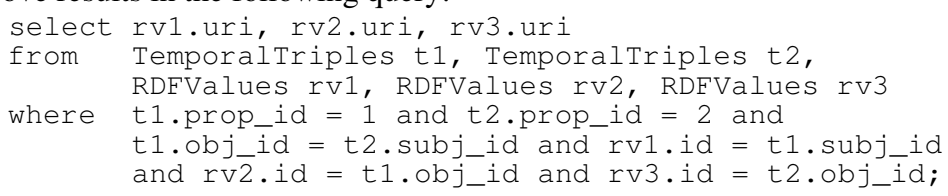

Spatial operators are implemented by augmenting the base graph pattern query in start(). For the spatial_extent operator, we add an additional join with the SpatialData table to retrieve the SDO_GEOMETRY object corresponding to the spatial_variable parameter. In the case of optional result filtering, we need to modify the where clause so that we filter the spatial features from SpatialData according to the input spatial feature and spatial relation. This is done by adding the appropriate sdo_relate or sdo_within_distance predicate available in Oracle Spatial. For example, given the query spatial_extent (..., sdo_geometry $(\ldots)$, 'geo_relate (inside)'), we would modify the query as follows: where ... and sdo_relate (SpatialData.shape, sdo_geometry (...), 'mask=inside') = 'true',

For the spatial_eval operator, we implement what is essentially a nested loop join (NLJ) using the basic spatial_extent and filtered spatial_extent operators. We first construct and execute a basic spatial_extent query in the start() routine. Next, in the fetch () routine, we consume a row from the spatial_extent query and then construct and execute the appropriate filtered spatial_extent query using the second pair of graph pattern and spatial variable parameters and the spatial relation parameter. This is repeated until all rows in the outer spatial_extent query are consumed. This NLJ strategy is needed to avoid an awkward query plan on what would be a very large single base query.

The implementation of the temporal operators does not translate directly to a SQL query. We must do some extra processing of the base query results in $f e t c h()$ to form a single time interval for each found graph pattern instance.

For the temporal_extent operator, we first augment the basic graph pattern query in start() to also select the start and end values for each temporal triple in the graph pattern instance. In the $f e t c h()$ routine, to compute the final temporal interval for each graph pattern instance, we examine the start and end times for each triple and select the earliest start and latest end $(R A N G E)$ or the latest start and earliest end (INTERSECT). In each case, we ensure that the resulting time interval is valid (i.e., start time less than end time) before including it in the result. When the optional filtering parameters are specified, we must perform additional checking of the found graph patterns to ensure they satisfy the filter condition. In addition to these extra computations in $\operatorname{fetch}()$, as an optimization, we augment the base query in $\operatorname{start}()$ with a series of predicates involving the start and end times of each statement in the graph pattern. This is done to filter the results as much as possible in the base query to reduce subsequent overhead in $f e t c h()$. To illustrate these additional predicates, consider the following temporal_extent query and corresponding base query:

select ...

from tabie (temporal_extent (' ( ?x on_crew_of ?y) (?y 


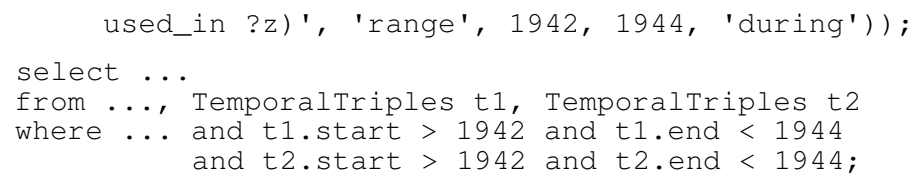

The implementation of the temporal_eval operator is similar to the implementation of spatial_eval. We first build a basic temporal_extent query involving the first pair of graph pattern and interval type parameters which is executed in the $\operatorname{start}()$ routine. Next, in fetch(), we consume a row from the basic temporal_extent query and execute an appropriate filtered temporal_extent query using the second pair of graph pattern and interval type parameters. This query uses the time interval from the current outer temporal_extent result and the inverse of the temporal relation parameter from the original temporal_eval query.

\section{Experimental Evaluation}

In this section, we describe the experimental evaluation of our spatial and temporal query operators. All experiments were conducted using Oracle 10g Release 2 running on a Red Hat Enterprise Linux machine with dual Xenon $3.0 \mathrm{GHz}$ processors and 2 GB of main memory. The database used an $8 \mathrm{~KB}$ block size and was configured with an sga_target size of $512 \mathrm{MB}$ and a pga_aggregate_target size of $512 \mathrm{MB}$. The times reported for each query are an average of 15 trials using a warm cache. Times were obtained by querying for systimestamp before and after query execution and computing the difference. Datasets and queries can be downloaded from http://knoesis.wright.edu/students/mperry/STData.html.

Dataset. Three synthetically generated datasets were used in our experiments. The datasets correspond to an ontology schema from the military domain that we created with the overall idea being to analyze historical entities and events of WWII. The ontology schema defined 15 class types and 9 property types. Each dataset was created in three phases. First we populated the thematic portion of the ontology. Second we added spatial information, and in the final step we generated temporal labels for the statements in the populated ontology.

To populate the thematic portion of the military ontology, we used the ontology population tool described in [31]. This tool inputs an ontology schema and relative probabilities for generating instances of each class and property type. Based on these probabilities, it generates instance data, which, in effect, simulates the population of the ontology. We generated three RDF datasets this way. The first contained 95,000 triples, the second contained 1.6 million triples and the third contained over 15 million triples (asserted and inferred statements). We integrated these military RDF graphs with the upper-level ontology described in [8] by adding a handful of rdfs:subClassOf statements to each RDF dataset.

To add spatial aspects to this dataset, we randomly assigned spatial features to each instance of Geometry in the ontology with uniform probability. We used year 2000 census block group boundary polygons from the US Census Bureau for the spatial features [32]. Differently-sized sets of contiguous US States were chosen in 
proportion with the ontology size. The total numbers of features for each dataset were $873 ; 9,352$ and 83,236 for the small, medium and large ontology, respectively.

The final phase of dataset generation assigned temporal labels to statements in the ontology. Temporal intervals were randomly assigned to each asserted instance statement. Start times and end times for each interval were randomly selected with uniform probability from two overlapping date ranges. We ensured that each interval was valid (i.e., start time earlier than end time) before adding it to the dataset.

Experiments. Our experiments are designed to characterize the overall performance of our approach with respect to (1) ontology size and (2) graph pattern complexity. For testing, B-Tree indexes were created on each column of the TemporalTriples table and on the value_id column of the SpatialData table, and an R-Tree index was created on the shape column of SpatialData. We also created two additional B-Tree indexes ( $p r o p \_i d$, subj_id, obj_id, start, end) and (prop_id, obj_id, subj_id, start, end) on the TemporalTriples table. For the 15 million triple dataset, the physical size of the TemporalTriples table was $642 \mathrm{MB}$, and the inferencing procedure took 1 hour and 31 min. to execute, which compared with 1 hour and 11 min. for Oracle RDFS rules index creation. The SpatialData table was $47 \mathrm{MB}$ in size.

Query Execution Time. Table 1 summarizes the results of our experimentation with respect to ontology size.

Table 1. Experimental results for query execution time with respect to ontology size

\begin{tabular}{llllllll}
\hline $\begin{array}{l}\text { Operator } \\
\text { (Exp. \#) }\end{array}$ & \multicolumn{2}{c}{$\begin{array}{l}\text { Graph Pattern Type } \\
\text { \# Vars }\end{array}$} & \# Triples & Queries & $\begin{array}{l}\text { Avg. } \\
\text { Result } \\
\text { Size }\end{array}$ & \multicolumn{3}{c}{$\begin{array}{l}\text { Avg. Execution Time for } \\
\text { each ontology (ms) } \\
\text { Small }\end{array}$} & Medium & Large \\
\hline T-Ext (1) & 4 & 3 & 4 & N/A & 394 & 390 & 385 \\
$(2)$ & 3 & 3 & 5 & 221 & 22 & 32 & 48 \\
S-Ext (3) & 4 & 3 & 3 & N/A & 360 & 350 & 365 \\
(4) & 3 & 3 & 3 & 100 & 22 & 30 & 67 \\
T-Filter(5) & 4 & 3 & 4 & 312 & 157 & 345 & 714 \\
S-Filter (6) & 4 & 3 & 3 & 331 & 173 & 192 & 374 \\
T-Eval(7) & $2 / 2$ & $2 / 2$ & 3 & 129 & 414 & 411 & 437 \\
& $2 / 3$ & $3 / 3$ & 3 & 220 & 306 & 195 & 268 \\
S-Eval (8) & $2 / 2$ & $2 / 1$ & 3 & 244 & 343 & 467 & 485 \\
& $2 / 2$ & $2 / 3$ & 3 & 209 & 251 & 385 & 457 \\
\hline
\end{tabular}

Experiments 1 through 4 were designed to test the general scalability of basic temporal_extent and spatial_extent queries. Experiments 1 and 3 measured the response time (i.e., time to return the first 1000 rows of results) for a very unselective query. Our unselective graph patterns consisted of 3 triples and 4 variables. For each triple in the pattern a constant URI was given for the property, and the subject and object were left as variables. We used 4 different graph patterns for temporal_extent with an INTERSECT type query in each case. For spatial_extent, 3 different graph patterns were used. In each case, the DBMS uses a nested loop joion (NLJ) strategy for evaluating the base query which results in response times which are essentially constant across each dataset as the execution time of a NLJ usually grows in proportion with the result set size. Experiments 2 and 4 are designed to measure 
scalability for a very selective graph pattern. For experiment 2, we used 5 different graph patterns consisting of 3 triples and 3 variables. For experiment 4, we used 3 different graph patterns with 3 triples and 3 variables. The graph patterns are of the same basic form as the previous experiment except we replace one of the variables in the subject or object position with a constant URI. This restricts the nodes in the resulting graph pattern instance instead of just the edges, providing a much more selective query. In each case, query execution time increases slightly as the ontology size increases, which is a consequence of scanning larger indexes during query evaluation and querying a larger SpatialData table.

In experiment 5, we measured the scalability of the temporal_extent operator using optional filtering with respect to dataset size. For these tests, we used very unselective graph patterns in combination with very selective temporal conditions. Note that this represents a worst case scenario for temporal_extent. Because we only store the temporal labels for single triples in the $\mathrm{DB}$, we can only index these single triples. The temporal labels for graph pattern instances are constructed during query evaluation and therefore cannot be indexed. We must apply the temporal filter to each graph pattern instance as it is being constructed, which can potentially lead to very large intermediate result sets because in many cases we cannot exclude a graph pattern from the results until its time interval has been fully constructed. Our experiments show an increase in execution time which is roughly linear with respect to ontology size which reflects the growth of intermediate results processed during the query. Each query used the INTERSECT option and either a before, after or during temporal relation.

In experiment 6, we measured the performance of spatial_extent using the optional filtering capability as dataset size increases. As with experiment 5, we combined a low selectivity graph pattern with a highly-selective spatial predicate. We used three different queries. The first retrieved results which were within a short distance of a point; another retrieved results which were covered by an input polygon, and the final query retrieved results which intersected with an input polygon. The results show that spatial_extent with filter scales better than its temporal counterpart because we can effectively index the spatial features and quickly reduce the search space using the spatial index. The execution time increases because larger indexes must be scanned when evaluating the graph pattern.

Experiment 7 illustrates the scalability of selective temporal_eval queries. For this test, we used selective graph patterns for both the LHS and RHS input patterns. We varied the constant URIs in the graph pattern and the temporal condition so that the result set sizes were constant across each dataset. The results show that execution time is roughly constant across each dataset with variations resulting from slight differences in the number of query restarts required in $f e t c h()$ and the selectivity of the graph patterns used. Each query used the INTERSECT option and either a before, after, during or any interact temporal relation. 

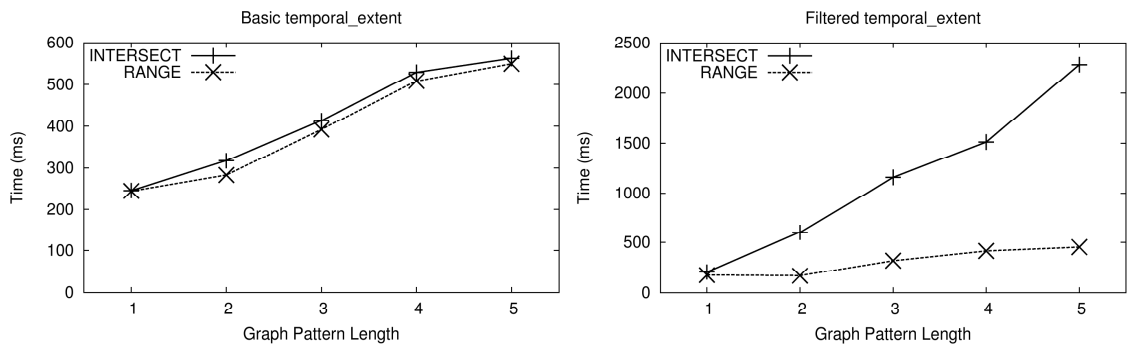

Fig. 3. Scalability of temporal operators with respect to graph pattern size

Experiment 8 characterizes the performance of selective spatial_eval queries as the dataset size increases. Again, we used selective graph patterns for both the LHS and RHS pattern and varied the constant URIs and spatial predicates so that result set size was consistent across each dataset. The results show that execution time grows slightly as ontology size increases, which is a result of scanning larger indexes and querying a larger spatial dataset.

Our next experiments were designed to test the scalability of the temporal_extent operator as the graph pattern size increased. We elected to present experimental results for only temporal queries due to space limitations, and, because temporal processing is less efficient than spatial processing in our scheme, these numbers should represent an upper bound. All queries in these tests were run against the 15 million triple dataset. The graph on the left side of Fig. 3 shows the response time (first 1000 rows) of basic temporal extent queries (INTERSECT vs. RANGE) for low selectivity graph patterns of increasing length. The times are the mean of 4 different queries for a given length. Each graph pattern has a constant URI in each predicate position and variables in each subject and object position. The results show that response time scales roughly linearly with graph pattern size. More processing time is required for INTERSECT because of extra join conditions needed to ensure valid time intervals. The graph on the right side of Fig. 3 shows the execution time for filtered temporal_extent queries using unselective graph patterns and selective temporal predicates. The idea behind this experiment was to bound the execution time for filtered temporal_extent queries. In some circumstances, our filtering optimization in the base query can only place weak conditions on the temporal properties of each triple in the result. For example, using INTERSECT and during $[x, y]$, we can only enforce that each triple does not end before $x$ or start after $y$. In contrast, using $R A N G E$ and during $[x, y]$ we can enforce that each triple both starts after $x$ and ends before $y$, which completely filters any unmatching graph patterns. The graph in Fig. 3 (right) shows the execution times for each scenario. Each value is the average of four different queries of that type. We can see that performance using the worst-case scenario scales much worse than the best case, but the growth is still roughly linear. The temporal predicates were increasingly selective as the pattern length increased to keep result set size constant for each query. We should note that we needed to pass a FIRST_ROWS hint to the query optimizer to avoid a query plan containing a full table scan in the case of the RANGE query (we provide an option to communicate this hint with our implementation). 


\section{Conclusions}

This paper discussed an approach for realizing spatial and temporal query operators for Semantic Web data. Our work was motivated by a lack of support for spatial and temporal relationship analysis in current semantic analytics tools. Spatial and temporal data is critical in many analytical applications and must be effectively utilized for semantic analytics to reach its full potential. Our approach built upon existing support for storage and querying of RDF data and spatial data in Oracle DBMS. A set of experiments using a synthetic RDF dataset of over 15 million triples showed that our implementation exhibited good scalability for a fairly large populated ontology. Basic temporal_extent and spatial_extent queries were quite fast in all circumstances. The worst performance was seen with filtered temporal_extent queries using low selectivity graph patterns with highly selective temporal predicates. However, the resulting execution times were quite manageable.

A possible limitation of this work is that Oracle Semantic Data Store does not support incremental maintenance of RDFS rules indexes. Consequently, our indexing scheme inherits this limitation. However, incremental maintenance of a materialized set of inferred triples upon updates of asserted triples is possible (e.g., [33]), and existing algorithms could be extended to incorporate temporal information.

In the future, we plan investigate this incremental maintenance issue and to perform further testing using other ontologies populated with both real and synthetic data. We also plan to investigate extensions of the SPARQL query language which support the types of operations discussed in this paper.

\section{References}

1. RDF. http://www.w3.org/RDF/

2. Anyanwu, K., Sheth, A.P.: $\rho$-Queries: Enabling Querying for Semantic Associations on the Semantic Web. In: 12th Int'1 World Wide Web Conf., Budapest, Hungary (2003)

3. Ramakrishnan, C., et al.: Discovering Informative Connection Subgraphs in Multi-relational Graphs. SIGKDD Explorations. 7(2), 56-63 (2005)

4. Aleman-Meza, B., et al.: Semantic Analytics on Social Networks: Experiences in Addressing the Problem of Conflict of Interest Detection. In: 15th Int'1 World Wide Web Conf., Edinburgh, Scotland (2006)

5. Mukherjea, S., Bamba, B.: BioPatentMiner: An Information Retrieval System for BioMedical Patents. In: 30th Int'l Conf. on Very Large Data Bases, Toronto, Canada (2004)

6. Kochut, K., Janik, M.: SPARQLeR: Extended Sparql for Semantic Association Discovery. In: 4th European Semantic Web Conf., Innsbruck, Austria (2007)

7. Pelekis, N., et al.: Literature Review of Spatio-Temporal Database Models. The Knowledge Engineering Review. 19(3), 235-274 (2004)

8. Perry, M., Hakimpour, F., Sheth, A.P.: Analyzing Theme, Space and Time: an Ontologybased Approach. In: 14th ACM Int'l Symposium on Geographic Information Systems, Arlington, VA (2006)

9. Gutierrez, C., Hurtado, C., Vaisman, A.: Temporal RDF. In: European Conf. on the Semantic Web, Heraklion, Crete, Greece (2005)

10.Brickley, D., Guha, R.V.: RDF Vocabulary Description Language 1.0: RDF Schema, W3C Recommendation. 2004: http://www.w3.org/TR/rdf-schema/ 
11.Hayes, P.: RDF Semantics. http://www.w3.org/TR/rdf-mt/

12.Yuan, M.: Wildfire Conceptual Modeling for Building GIS Space-Time Models. In: GIS/LIS, Pheonix, AZ (1994)

13.Yuan, M.: Modeling Semantical, Temporal and Spatial Information in Geographic Information Systems. In: Craglia, M., Couclelis, H. (eds.) Geographic Information Research: Bridging the Atlantic. pp. 334-347. Taylor \& Francis (2006)

14.Worboys, M., Hornsby, K.: From Objects to Events: GEM, the Geospatial Event Model. In: Geographic Information Science: 3rd Int'1 Conf., Adelphi, MD (2004)

15.Prud'hommeaux, E., Seaborne, A.: SPARQL Query Language for RDF. http://www.w3.org/TR/rdf-sparql-query/

16.Karvounarakis, G., et al.: RQL: A Declarative Query Language for RDF. In: 11th Int'1 World Wide Web Conf., Honolulu, HI (2002)

17.Sintek, M., Decker, S.: TRIPLE - A Query, Inference, and Transformation Language for the Semantic Web. In: 1st Int'1 Semantic Web Conf., Sardinia, Italy (2002)

18.Souzis, A.: RxPath Specification Proposal. 2004: http://rx4rdf.liminalzone.org/RxPathSpec

19.Wilkinson, K., et al.: Efficient RDF storage and retrieval in Jena2. In: VLDB Workshop on Semantic Web and Databases, Berlin, Germany (2003)

20.Alexaki, S., et al.: On Storing Voluminous RDF Descriptions: The Case of Web Portal Catalogs. In: 4th Int'1 Workshop on the Web and Databases, Santabarbara, CA (2001)

21.Chong, E.I., et al.: An Efficient SQL-based RDF Querying Scheme. In: 31st Int'1 Conf. on Very Large Data Bases, Trondheim, Norway (2005)

22.Kammersell, W., Dean, M.: Conceptual Search: Incorporating Geospatial Data into Semantic Queries. In: Terra Cognita - Directions to the Geospatial Semantic Web, Athens, GA (2006)

23.Tanasescu, V., et al.: A Semantic Web GIS based Emergency Management System. In: Int'1 Workshop on Semantic Web for eGovernment, Budva, Montenegro (2006)

24.Egenhofer, M.J.: Toward the Semantic Geospatial Web. In: 10th ACM Int'l Symposium on Advances in Geographic Information Systems, McLean, VA (2002)

25.Jones, C.B., et al.: The SPIRIT Spatial Search Engine: Architecture, Ontologies, and Spatial Indexing. In: 3rd Int'l Conf. on Geographic Information Science, Adelphi, MD (2004)

26. Oracle Spatial Resource Description Framework (RDF) 10g Release 2. http://downloadeast.oracle.com/docs/cd/B19306_01/appdev.102/b19307/toc.htm

27.Oracle Spatial User's Guide and Reference 10g Release 2. http://downloadeast.oracle.com/docs/cd/B19306_01/appdev.102/b14255/toc.htm

28.Hakimpour, F., et al.: Data Processing in Space, Time and Semantics Dimension. In: Terra Congita - Directions to the Geospatial Semantic Web, Athens, GA (2006)

29.Open GIS Consortium: Open GIS Simple Feature Specification for SQL. 1999: http://portal.opengeospatial.org/files/?artifact_id=829

30. Oracle Database Data Cartridge Developer's Guide, 10g Release 2. http://downloadeast.oracle.com/docs/cd/B19306_01/appdev.102/b14289/toc.htm

31.Perry, M.: TOntoGen: A Synthetic Data Set Generator for Semantic Web Applications. AIS SIGSEMIS Bulletin. 2(2), 46-48 (2005)

32.U.S. Census $2000 \quad$ Cartographic Boundary Files. http://www.census.gov/geo/www/cob/bg2000.html

33.Volz, R., Staab, S., Motik, B.: Incrementally maintaining materializations of ontologies stored in logic databases. Journal on Data Semantics. 2, 1-34 (2005) 